\title{
Aspectos bioéticos de la asistencia al drogodependiente
}

\author{
Morera Pérez, B. \\ Jefe Clínico del Área de Media Estancia. Hospital Aita Menni Arrasate-Mondragón. Guipúzcoa. \\ Enviar correspondencia a: \\ Blanca Morera Pérez. Hospital Aita Menni. Barrio Gesalibar 15. 20509 Arrasate-Mondragón. Tfno: 943-794411.
}

\section{RESUMEN}

Uno de los objetivos esenciales de la Bioética es incorporar los valores, junto a los hechos, en las decisiones propias de aquellas disciplinas científicas relativas a la Vida. En ámbitos asistenciales la Bioética pretende desvelar y superar aquellos conflictos de valores derivados de una interrelación en la que se implican sujetos individuales y / o grupos sociales.

Las drogodependencias representan un campo idóneo para este análisis porque suscitan conflictos de valores a niveles muy diversos. Constituyen un problema complejo en el que interaccionan intereses políticos, económicos, sociales, culturales y sanitarios.

En el ámbito asistencial, la reflexión bioética, tanto en su momento deontológico como teleológico, aporta una perspectiva complementaria a la terapéutica. Puede facilitar el abordaje de numerosos problemas, que van de la identificación del marco conceptual a la detección de los criterios de elección entre distintos programas (libres de drogas, mantenimiento o reducción de daños).

Un acercamiento progresivo a aspectos como delimitación y protocolización de buenas prácticas clínicas, valoración de la competencia, desarrollo de la autonomía individual, equidad en la distribución de recursos asistenciales o implementación de medida de beneficencia, entre otros aspectos, puede ayudar a comprender, integrar y superar algunos problemas de las drogodependencias desde una perspectiva asistencial.

Palabras clave: drogas, drogodependencia, aspectos éticos, principios éticos, tratamiento.

\section{SUMMARY}

One of the most important aims in Bioethics is bringing together values and facts in decision - making strategies in scientific disciplines concerning Life. In clinical settings, increasing conflicts have arisen in relation to values involved in relationships between subjects or subjects and social groups. Bioethics attempts to display these conflicts, first and, secondly, overcome them.

Drug addiction does represent a suitable field for bioethical analysis for it implies value conflicts in many different levels. It is a complex problem where political, economic, social, cultural and clinical interests can be identified.

In managing settings Bioethics provides complementary perspectives to treatment strategies, both through deontological and teleological reflections. Ethical issues can facilitate reappraisal of drug addiction-related problems, which vary from defining conceptual background or treatment frameworks to establishing criteria in therapeutic program assignation (when free-drug programs and programs of maintenance and risk management are available).

Given that difficult task, a progressive approach to ethical issues as good clinical practices, protocols, capacity / competence assessment, promotion of individual autonomy, equitable distribution of resources and services and implementation of beneficent strategies, may help to understand and go beyond drug addiction and get addicted subjects treated and integrated.

Key words: drug, drug addiction, ethical issues, ethical principles, treatment.

\section{BIOÉTICA: CONCEPTOS PRELIMINARES}

$\mathbf{L}$ a Bioética puede definirse como "el estudio interdisciplinar (transdisciplinar) orientado a la toma de - decisiones éticas de los problemas planteados a los distintos sistemas éticos por los progresos médi- cos y biológicos, en el ámbito microsocial y macrosocial, micro y macroeconómico, así como su repercusión en la sociedad y su sistema de valores, tanto en el momento presente como futuro". Son elementos constitutivos de la Bioética su carácter secular, la metodología basada en el diálogo, el reconocimiento de la autonomía de las ciencias, los argumentos basa- 
dos en el razonamiento (evitando criterios de autoridad), la asunción del carácter "provisional" de sus conclusiones y la preocupación por los problemas de la población, los recursos y el medio ambiente.(1)

La Bioética nace ligada a esa ancestral preocupación del ser humano sobre lo que está bien y lo que está mal, sobre lo que se debe o no se debe hacer, en definitiva sobre las conductas y los valores. Tiene una función "mediadora" entre las éticas de código único (en las que los valores morales se consideran valores objetivos, cognocibles por todos y exigibles por igual a todas las personas) y las éticas de código múltiple (nacidas desde el reconocimiento, para cada sujeto, de la capacidad de autogobierno, también moral, y de la autodeterminación de los individuos).(2) La Bioética pretende buscar, desde el consenso, criterios intersubjetivos que la sociedad en su conjunto pueda aceptar como "valores asumibles".

En este marco teórico, la bioética clínica no es sino ética aplicada a un ámbito específico como es la asistencia sanitaria. En esta vertiente, que ha tenido un desarrollo muy importante en las últimas décadas, la ética se desarrolla ligada fundamentalmente a tres hechos: Ios continuos avances de la medicina, que condicionan una complejidad progresiva en las decisiones clínicas y en las condiciones de asistencia sanitaria; el desarrollo de los derechos humanos que devuelve al sujeto el protagonismo en su proceso de enfermedad y en las decisiones que le afectan; y la generalización, en nuestro medio, de la asistencia sanitaria, que nos obliga a reevaluar la relación recursos-necesidades.

El aspecto más pragmático de la bioética viene constituido por la identificación de los conflictos de valores que subyacen en las decisiones asistenciales, de forma que el análisis de los hechos objetivos, a lo que estamos acostumbrados desde una formación científica, pueda complementarse con el análisis de los valores en riesgo.

A lo largo de este trabajo trataremos de desarrollar cómo puede darse una visión complementaria, entre otras, al tema de las drogodependencias, describiendo, al menos, las cuestiones y los conflictos que se plantean desde una perspectiva bioética en la atención al drogodependiente.

Antes de abordar este tema, y para completar esta breve introducción a conceptos bioéticos básicos, conviene sin embargo hacer algunas consideraciones de corte metodológico. Estas consideraciones pueden resultar ociosas a quienes ya hayan tenido contacto con esta disciplina, pero resultan imprescindibles para quienes se acerquen por primera vez al lenguaje bioético. Como en otras disciplinas de conocimiento, los marcos teóricos y las metodologías empleadas en Bioética han sido variadas y no excluyentes. En este trabajo vamos utilizar, fundamentalmente, la metodo- logía propuesta por el Profesor Diego Gracia(2), eminente teórico de la Bioética en nuestro país, metodología que consideramos idónea en el ámbito de la bioética clínica por su utilidad práctica y la comprensibilidad de los conceptos que maneja, tan cercanos a nuestro propio bagaje cultural.

Desde esta metodología se propone como fundamentación un sistema de referencia de carácter formal, que se expresa en dos principios: un principio ontológico formulado como "el ser humano es persona y, en cuanto tal, tiene dignidad y no precio" y un principio ético formulado como "en tanto que personas, todos los seres humanos son iguales y merecen la misma consideración y respeto". La metodología propone que este sistema de referencia - y por tanto ambos principios - sea exigible siempre y de forma universal, sin excepciones posibles.

Sobre este sistema de referencia se articulan los dos momentos del análisis bioético(3): el momento deontológico ("a priori" o "vis a tergo"), en el que los cursos de acción posibles - las decisiones asistenciales, en nuestro caso -se someten a evaluación desde los principios éticos (que comentaremos enseguida), y el momento teleológico ("a posteriori" o "vis a fronte") en el que se analizan las consecuencias en caso de que dichos cursos de acción se utilizaran hipotéticamente.

Los principios éticos propuestos para la bioética clínica son cuatro(4):

- El principio de no-maleficencia: ligado estrechamente al "primum non nocere" hipocrático, hace referencia al deber del clínico de no dañar al paciente, y por tanto de realizar adecuadamente su trabajo profesional. Es un principio que trata de la obligación de establecer las "buenas prácticas clínicas" y aplicarlas en cada caso. Este valor se adscribe fundamentalmente a los profesionales sanitarios y asistenciales. Las referencias se explicitan en dos conceptos: "la formación y la indicación". Por debajo de él se sitúa la impericia, la negligencia y el abandono.

- El principio de justicia que establece la obligación de proporcionar a todos los sujetos las mismas oportunidades -" a cada uno según sus necesidades"- en el orden de lo social, no discriminando, segregando o marginando. Aunque este valor implica de forma preferente a políticos y gestores, que se ven por él obligados a procurar la igualdad de oportunidades ante los recursos sanitarios y a garantizar la realización del derecho a la dignidad a través de quienes intervienen en la asistencia sanitaria, alcanza en sus propios niveles a los profesionales, tanto en los aspectos de gestión que pueden competerles como en su calidad de miembros de una sociedad civil capaz de reclamar condiciones de justicia para sí y para otros.

- El principio de autonomía que exige respetar la libertad del otro para decidir y disponer de sí mismo, 
estableciendo lo que es bueno para él en función de su individual y personal proyecto de vida, reconociendo en todo sujeto "a priori" la capacidad para ello. El eje práctico de este valor ha sido el consentimiento informado, proceso en el que comparten protagonismo pacientes y profesionales, el primero en el ejercicio del derecho, y el segundo promoviendo que la situación global permita la elección libre del paciente.

- Finalmente, el principio de beneficencia, que exige hacer el bien a quien nos lo pide, o a quien no pudiendo pedirlo lo precisa, pero respetando siempre "el criterio de bien" del propio beneficiario (esto es, respetando su propio proyecto vital), a quien se representa cuando él mismo no puede hacerlo. Este valor residió hasta hace muy poco tiempo en los profesionales formados en una práctica paternalista, y es ahora un bien compartido por profesionales (que deben evaluar la competencia) y los allegados que son titulares de las Ilamadas "decisiones de sustitución", decisiones que deben implementarse cuando los sujetos carecen de autonomía.

Todos estos principios cumplen los criterios de intersubjetividad que Sir David Ross estableció para los llamados "deberes prima facie", por lo que se considera que la mejor decisión asistencial es aquella que respeta todos ellos. Cuando ello no es posible y se produce conflicto entre principios se hace necesaria una jerarquización que Diego Gracia(2) clarifica estableciendo una "ética de mínimos" o de primer nivel, donde engloba los principios de no-maleficencia y justicia, que se califican como "deberes perfectos", esto es, deberes que, de ser lesionados, comprometen un derecho básico de las personas; por tanto, existe acuerdo general en cuanto a que su cumplimiento debe ser exigido. Los principios de autonomía y beneficencia quedan englobados en el segundo nivel, considerado de "ética de máximos", que comprende unos máximos morales o deberes imperfectos que protegen los valores y proyectos vitales de cada individuo de forma particular, en forma de actos que cada uno elige para sí mismo pero que no puede imponer a otros, por lo que, aún debiendo respetarse, no pueden ser exigidos de forma absoluta si ello requiere dañar o discriminar.

\section{DROGODEPENDENCIAS: ¿ES POSIBLE UN ACER- CAMIENTO BIOÉTICO?}

Añadido al hecho inapelable de que en las relaciones humanas (y las relaciones asistenciales no son sino un tipo de ellas) no existen actos éticamente neutros(5) las drogodependencias representan, por sus propias características, un campo idóneo para el acercamiento bioético, y ello por varias razones: es un fenómeno protagonizado por seres humanos (por tanto tratado por las ciencias de la vida en todo su sentido) que, por una parte, tiene una relevancia epidemiológica que resulta incuestionable (a nivel local, nacional e internacional) y que, por otra parte, implica factores muy diversos (económicos, culturales, sociales, políticos, sanitarios, etc. ). Esta enorme complejidad condiciona que los conflictos de valores se produzcan en situaciones muy variadas. A pesar de ello, o quizá por ello, las drogodependencias han recibido poca atención en comparación con otros ámbitos sanitarios por parte de los bioeticistas. Otras razones que se esgrimen para justificar este fenómeno de relativo distanciamiento tienen que ver con la evidencia de que la Bioética Clínica ha estado casi exclusivamente centrada durante su corta historia en el impresionante desarrollo tecnológico de la Medicina(6) Este desarrollo ha condicionado múltiples ámbitos de conflicto en las decisiones sanitarias, la mayoría de ellas ligadas a las medidas extraordinarias que se han generalizado en el ámbito hospitalario (indicación o inactivación de medidas de soporte vital, intervenciones quirúrgicas en situaciones límite, reanimación, medidas extraordinarias en relación a neonatos o en el final de la vida, concepto de muerte, etc.) y a los avances de determinadas líneas de investigación genética y farmacológica. Sólo cuando la bioética se ha empezado a hacerse "familiar" entre los profesionales se ha empezado a avanzar en el estudio de los conflictos éticos de diferentes especialidades, de diferentes patologías y de diferentes ámbitos de tratamiento. En esta tesitura nos encontramos ahora, en el momento de identificar conflictos más que de dar soluciones definitivas, de establecer marcos de acuerdo más que consensos irrevocables, en definitiva, en el momento de conocer los hechos y los valores en liza para poder, posteriormente, proponer cursos de acción.

No cabe duda que el primer problema que encontramos cuando nos acercamos a las drogodependencias es la fuerte carga emocional e ideológica que conlleva, lo que se traduce en posicionamientos dilemáticos casi a cualquier nivel en que nos ubiquemos. Estas opciones "límite" se traducen en la confrontación de posturas como legalización versus penalización, programas libres de droga versus programas de sustitución, marginalización versus normalización, etc. Estos planteamientos, difícilmente reconciliables en algunas ocasiones, no facilitan el debate ni el consenso y además olvidan en muchos casos la extraordinaria complejidad del hecho al que nos enfrentamos(7).

Parte de este problema es, en el caso de las drogodependencias, el conflicto que se plantea entre la sociedad y el individuo(8,9), conflicto que contiene en sí mismo la clásica dialéctica vida - libertad: ¿Es lícito que un sujeto utilice su propia libertad en su perjuicio? ¿Dónde se establecen los límites entre la ley y la autonomía individual? ¿Es moralmente aceptable que el 
derecho de conciencia y la libertad para el autogobierno sancionen la elección del autoabandono o por el contrario éste resulta una excepción injustificable a dicho derecho? ¿Debe la sociedad interferir en estos casos? ¿Hasta dónde puede actuar la sociedad en la prevención del daño de terceros? ¿Tiene obligaciones la sociedad con un sujeto que no puede cumplir sus responsabilidades sociales por una elección libre? ¿Hasta dónde puede asumir la sociedad costes sociales y sanitarios derivados de decisiones libres y autónomas?. Superaría con mucho el objetivo de esta presentación el tratar de responder, siquiera parcialmente, a estas preguntas y a otras muchas que podrían plantearse. El análisis bioético de las relaciones sociedad - individuo en relación a las drogodependencias sin duda requiere, y seguirá haciéndolo en el futuro, el intercambio de múltiples niveles de conocimiento e información, muchas deliberaciones, muchos encuentros y desencuentros, antes de conseguir consensos que, probablemente, seguirán sorprendiéndonos por su caducidad(9). Sin embargo este conflicto individuo - sociedad resulta relevante a nuestro objetivo, esto es el acercamiento a lo aspectos bioéticos del drogodependiente, porque las drogodependencias confrontan al profesional asistencial a la existencia de dos niveles en estrecha interacción: un micronivel donde se produce la interacción individual profesional-drogodependiente, y donde el adicto se presenta como un ser humano que solicita ayuda, padece un trastorno o tiene demandas farmacológicas específicas, y un macronivel donde las drogodependencias se constituyen en tema legal y social, con implicación de intereses políticos, financieros y con actividades criminales asociadas. Para algunos autores el tratamiento de los adictos se va a ver afectado significativamente, y de hecho así parece confirmarse en la práctica, por el acercamiento social que se realice al problema adictivo, que condiciona a menudo desde la propia ideología del profesional, a los medios terapéuticos disponibles y a la injerencia de aspectos no sanitarios en las políticas sanitarias y en la práctica asistencial cotidiana.(10)

Debatir si el profesional debe mantener en la relación terapéutica y en la atención individualizada su relación con el drogodependiente, o si debe participar como coagente social en el manejo global del hecho "drogodependencias" en línea con las directrices que acuerde la sociedad, más allá de su alianza o del contexto asistencial, supondría un nuevo y profundo debate bioético. A fin de definir un marco más específico, y sin reconocer en ello un cierto artificio teórico en la descripción de los conflictos bioéticos de la drogodependencia, asumiremos la asistencia como un hecho mayoritariamente ligado a la práctica clínica y ajeno a las conceptualizaciones sociales.

La necesidad de acotar nuestro marco nos lanza de lleno al segundo gran problema (también bioético) que plantea la asistencia al drogodependiente: ¿Qué vamos a considerar drogodependencia? ¿qué vamos a considerar droga?. Resulta un conflicto de primera magnitud a la hora de realizar un análisis bioético el acotar el campo de estudio y ello supone una tarea tan difícil como establecer con cierta claridad los límites de la asistencia al drogodependiente. Se trata de saber quienes son los sujetos que definimos como drogodependientes y que tipo de problemas definimos como drogodependencias y por lo tanto a quiénes y a qué vamos a dirigir las medidas asistenciales que aquí pretendemos analizar desde una perspectiva bioética.

Una vez más debemos partir de conceptos establecidos por consenso para tratar de unificar el lenguaje. Reconocemos que el concepto "droga" se encuentra en permanente dificultad para ser definido(11); se trata de un concepto "demonizado" que, sin embargo, no hace sino calificar el conjunto de sustancias químicas que, introducidas en un organismo humano, son capaces de modificar su funcionalismo. Caben en esta definición fines tan variados como la terapéutica, la búsqueda del placer y de la experiencia mística, el alivio de determinados efectos, síntomas o sensaciones, la estimulación o la sedación, por citar unos pocos. Se trata por tanto de una práctica universal en la que han confluido sujetos, sustancias y usos muy diferentes. No obstante en nuestro tiempo han ido conformándose modelos culturales que oponen el uso de sustancias socialmente aceptadas ("drogas legales") como son en nuestro medio el alcohol, el tabaco, y algunos fármacos, entre otros, al uso de sustancias inaceptables ("drogas ilegales") donde se reúnen sustancias de efectos y poder adictivo tan variables como la heroína y otros mórficos, la cocaína, el cannabis o los derivados anfetamínicos sintéticos, por citar algunos(12). Resulta difícil explicar, sin recurrir a factores de índole política, económica, social y sanitaria, la asignación de sustancias a uno u otro grupo, que históricamente han recibido una aceptación social cambiante.

Para el abordaje de las drogodependencias se identifican, longitudinal y transversalmente en el tiempo, al menos tres modelos(13) que, entremezclados y subclasificados, hacen casi innumerables las perspectivas posibles. Por un lado existe un modelo penal, donde las medidas de orden jurídico adquieren el máximo protagonismo, siendo los objetivos la represión del consumo y de la disponibilidad. En este modelo la droga representa casi exclusivamente un objeto de delito y los usuarios quedan estigmatizados y criminalizados, en base a la consecución del "bien común". De ahí que los recursos se orienten a implementar acciones policiales y judiciales, al control de tráfico de sustancias y a la sanción del consumo. Es un modelo que refuerza el estereotipo drogas legalesdrogas ilegales. En segundo lugar puede hablarse de 
un modelo médico, donde el drogadicto es un enfermo, situación que ha sido formulada desde submodelos morales, de aprendizaje, de trastorno, de automedicación, sociales, etc., en función de los factores etiológicos contemplados. Finalmente existe un modelo sociocultural, que reubica el fenómeno en un contexto antropológico y etnográfico.

Cuando hablamos de asistencia no cabe duda que nos situamos en el segundo modelo, aunque abogando por modelos integrativos que tratan de establecer puntos de conexión entre los diferentes submodelos, de una manera no excluyente. A efectos de nuestro análisis la dependencia se va a entender en los términos que expresa clasificaciones internacionales como la décima edición de la Clasificación Internacional de Enfermedades de la OMS (CIE-10), esto es, "como el conjunto de manifestaciones fisiológicas, comportamentales, y cognoscitivas, en el cual el consumo de una droga, o de un tipo de ellas, adquiere la máxima prioridad para el individuo, mayor incluso que cualquier otro tipo de comportamiento que, en el pasado, tuviera un valor más alto."

Como vemos, este planteamiento supera cualquier distinción entre drogas legales e ilegales, distinción irrelevante a efectos de definición del trastorno, y supera, por fin, la necesidad de un síndrome de abstinencia o de un fenómeno de tolerancia, para establecer la existencia del trastorno. Lo que en definitiva cualifica la adicción es que la sustancia se convierte en el fin (telos) de la vida física y psíquica del sujeto, sustituyendo cualquier otro proyecto vital, siendo consciente subjetivamente el sujeto de su compulsión a la búsqueda, obtención y consumo de dicha sustancia.

\section{CONFLICTOS Y DILEMAS ÉTICOS LIGADOS A LA ASISTENCIA}

No es posible en un trabajo tan reducido dar, siquiera, una panorámica completa de los conflictos y dilemas que una perspectiva bioética puede plantear en la asistencia al drogodependiente, por ello vamos a ceñirnos al acercamiento a algunos por la frecuencia con la que se presentan: el diagnóstico, la terapéutica, la competencia y las decisiones de sustitución. Como veremos surgen muchas más preguntas que respuestas.

\section{El diagnóstico del drogodependiente}

Un diagnóstico de drogodependencia no es en ningún caso un acto banal. Diagnosticar supone afirmar en un sujeto la existencia de un determinado trastorno, lo que supone una cierta forma de "etiquetaje".
Realizar un diagnóstico tiene dos objetivos fundamentales: en primer lugar el diagnóstico establece la naturaleza del trastorno que padece el sujeto y por tanto el diagnóstico resulta imprescindible para establecer la terapéutica, y por otra parte el diagnóstico "codifica" dicho trastorno en un lenguaje común a los diversos profesionales de forma que se hace posible la comunicación entre ellos, siempre con la finalidad de mejorar la asistencia(14). No obstante este proceso puede presentar problemas de relevancia bioética:

\section{Problemas técnicos en el establecimiento del diag- nóstico de drogodependencia}

La mayor parte de los problemas en el proceso de la realización de un diagnóstico de drogodependencia afectan al principio de no maleficencia. Asignar un diagnóstico de drogodependencia a un sujeto no dependiente "daña" al sujeto que recibe la etiqueta, no sólo por las consecuencias personales, familiares y sociales de dicho diagnóstico ( y no caben dudas de que la conducta adictiva es una de las conductas más explícitamente rechazadas en nuestra sociedad), sino porque en función de dicho diagnóstico se le pueden aplicar medidas terapéuticas innecesarias o que supongan riesgos físicos o psíquicos. Por el contrario el no-diagnóstico del trastorno puede privar al sujeto de la ayuda y los medios necesarios para su tratamiento o para la prevención de complicaciones asociadas a estos trastornos (problemas infecciosos, por ejemplo).

El diagnóstico de drogodependencia puede ser un diagnóstico incorrecto en función de que los criterios utilizados o los instrumentos diagnósticos no sean los adecuados, bien porque los medios no son idóneos, bien porque la persona que realiza el diagnóstico posee insuficiente formación para realizar el diagnóstico diferencial necesario o bien contempla de forma muy parcial todas las implicaciones del diagnóstico (situación física, gravedad de la adicción, trastornos psiquiátricos concomitantes, apoyos personales y sociales, etc.). Otro problema bioético importante esta representado por los que podríamos denominar "diagnósticos subjetivos o personalizados", etiquetajes que responden a criterios personales de determinados terapeutas pero que no resultan asumibles (y a veces no comunican información relevante) a otros profesionales.

Una tercera cuestión está representada por los "sobrediagnósticos": es bien cierto que la sociedad en su conjunto tiende a aceptar los criterios de los profesionales en cuanto a establecer qué conductas deben considerarse trastornos o enfermedades (y en ese sentido reciben ciertos estigmas pero también mayor tolerancia y mayores medios con fines terapéuticos) y qué conductas no. Los profesionales deben ser cuidadosos a la hora de psiquiatrizar, por motivos personales o ideológicos, conductas individuales. De 
ahí la importancia de no denominar "enfermedades o trastornos" a consumos ocasionales de carácter lúdico o pautas de uso de sustancias que no se corresponden con los criterios establecidos para las drogodependencias(7), pero también de señalar el trastorno, cuando existe, y así superar en parte el estigma de "inadaptados", "viciosos" o "antisociales" que arrastran muchos adictos.

Otro problema es el que los profesionales no establezcamos las enormes diferencias que existen entre los diferentes tipos de adicciones tanto desde el punto de vista de pronóstico, de riesgo personal y para terceros, de posibles complicaciones sanitarias, etc., con lo que ellos supone también de orientación de gastos sanitarios y, porque no decirlo, de gastos sociales.

Resulta imposible tratar en este abordaje inicial todos los conflictos éticos que surgen en relación a medios diagnósticos, pero no puedo menos que citar y hacer alguna consideración respecto al conflicto ético que representa la detección de tóxicos en el medio de trabajo, y que nos devuelve una vez más al conflicto de intereses sujeto individual-sociedad (en este caso empresas o terceros a riesgo) cuando se trata de las drogodependencias. Existe una bibliografía creciente sobre el tema que trata sus múltiples implicaciones $(15,16)$. Para el aspecto que a nosotros nos interesa, el ámbito asistencial, el foco de conflicto puede producirse cuando, generalmente en el contexto de la medicina de empresa o de las medicina de aseguración, no se clarifica la función "clínica" de la prueba, que de ser tal debe reunir todos los requisitos (indicación, adecuación de los medios, reserva y respeto a la dignidad del sujeto, confidencialidad de los resultados, etc.) propios de un acto sanitario(17).

\section{Problemas en el "uso" del diagnóstico de drogode- pendencia}

Otros conflictos ligados al diagnóstico tienen que ver con un uso no sanitario del diagnóstico de drogodependencia. Es moralmente inaceptable que el diagnóstico de drogodependencia suponga algún tipo de discriminación, marginalización o estigmatización y ello, cuando ocurre (lo que no resulta infrecuente) representa un conflicto de justicia claro, que debe ser conocido por quien realiza el diagnóstico y por quien lo conoce. Igualmente resulta ilícito utilizar un falso diagnóstico de drogodependencia para tener acceso a sustancias (con fines no terapéuticos), para acceder a recursos sociales o para evitar responsabilidades penales, todo lo cual puede considerarse un uso espurio de un acto clínico de indudable valor.

Como comentábamos al principio de este apartado el diagnóstico representa algo más que una etiqueta: supone el padecimiento de un trastorno con lo que conlleva de implicaciones terapéuticas, pronósticas y de afectación global de alguno o muchos aspectos de la vida del sujeto. El no poner toda esta información en manos del sujeto que lo padece, con la claridad y amplitud necesarias para que el sujeto pueda hacerse cargo de su propio problemas y tomar las decisiones con conocimiento adecuado sobre las implicaciones de las mismas, contraviene el derecho del sujeto (y por tanto afecta a la autonomía) pero también resulta maleficente desde el punto de vista del proceso diagnóstico y terapéutico, sustrayendo un elemento indispensable a la relación terapéutica. En este mismo orden de cosas el que el diagnóstico no sea tratado con la necesaria confidencialidad (que debe extremarse por su naturaleza especialmente "sensible") por difusión a terceros o por falta de custodia de los registros e historias clínicas, son conductas contrarias a la lex artis, y por tanto maleficentes, y constituyen en sí mismas conductas contrarias al derecho del paciente, que pone en nuestras manos los datos necesarios para el diagnóstico en la expectativa de que será tratado con la necesaria reserva y discreción.

\section{Algunas propuestas de cara al diagnóstico}

Algunas propuestas pueden emerger para tratar de superar estos conflictos: en primer lugar que el diagnóstico se realice con criterios e instrumentos fiables, a fin de obtener una certeza clínica razonable. Debe entenderse siempre que un diagnóstico de drogodependencia, sea cual sea la sustancia, y aunque existan diferencias entre ellas, supone un nivel de discriminación social importante, por lo que debe extremarse el evitar etiquetas cuando existan dudas razonables sobre los datos o sobre la gravedad, y por supuesto no deben darse opiniones subjetivas no científicamente fundadas. Debe garantizarse en este sentido la formación y adecuación de los profesionales que realicen el diagnóstico de drogodependencia, cuidando la necesaria actualización de los conocimientos, puesto que el conocimiento sobre el mundo de las drogas, y el propio mundo de las drogas evoluciona con rapidez y a menudo el drogodependiente requiere más de un diagnóstico concomitante. Resulta aconsejable utilizar criterios aceptados en la comunidad científica, aún siendo conscientes de sus muchas limitaciones, para facilitar la orientación terapéutica y la comunicación entre los profesionales. Las limitaciones de un etiquetaje según las clasificaciones internacionales debe complementarse con una descripción adecuada de todos aquellos datos complementarios al diagnóstico que constituyen el padecimiento individual del trastorno y que convierten al sujeto en lo que es, algo más que una enfermedad. También puede aportar mejoras en este proceso el acordar, en los casos en los que el diagnóstico se realice sobre las bases más débiles (criterios equívocos o inmaduros, argumentos insuficientes o parciales, diagnósticos con fuerte carga ideológica, etc.), propuestas de criterios diagnósticos que 
puedan sancionarse desde los profesionales implicados(18).

No debe evitarse el diagnóstico cuando éste sea claro, aunque teniendo siempre un cuidado excepcional en los aspectos relativos a la confidencialidad y, finalmente, debe evitarse prestar el diagnóstico a cualquier objetivo que no sea terapéutico, contando con el necesario consentimiento del sujeto para su comunicación a terceros, fuera o dentro del ámbito sanitario.

\section{EITratamiento de los drogodependientes}

Cuando hablamos de tratamientos, de aspectos asistenciales, en definitiva, no es posible realizar un abordaje global: existen muchas diferencias específicas entre lo que requiere un dependiente del alcohol para su tratamiento integral o las necesidades de un dependiente a opiáceos. En definitiva cada droga provoca, más allá del hecho general de la adicción, una serie de síntomas, signos, trastornos concomitantes y complicaciones, que les son propios. Tampoco todas las sustancias provocan el fenómeno adictivo en la misma forma, y no resulta posible establecer de forma fiable una jerarquía de toxicidad, hecho que depende de multitud de variables(19).

\section{La asignación al espacio sociosanitario dentro de la Psiquiatría}

Las drogodependencias han sido asignadas en su abordaje, aunque con cierta especificidad, a una especialidad médica, la Psiquiatría, cuya actuación complementan otras instituciones y profesionales. Como ésta, las drogodependencias se asignan a un espacio que se denomina "espacio sociosanitario", lo que supone la aceptación de al menos tres hechos en relación estrecha: que las drogodependencias responden a la interacción de múltiples factores etiológicos (entre ellos factores biológicos, psicológicos y sociales que consolidan un modelo biopsicosocial aún no bien demostrado pero que resulta plausible(20), que en el tratamiento se imbrinca la actuación de diferentes profesionales si hay que atender la multiplicidad de los problemas asistenciales planteados, y que esta misma complejidad asistencial requiere la coexistencia de diferentes abordajes. Esta asignación puede tener en ocasiones la dificultad de entremezclar el problema de la adicción y de los problemas derivados del consumo de la droga, problemas a menudo muy diferentes desde el punto de vista social y sanitario.

Esta situación tan compleja requiere un trabajo de equipo que ya encierra en sí mismo algunos problemas bioéticos como son: la falta de estructuración del trabajo de los diferentes profesionales implicados (que pueden impedir o dificultar la jerarquización de las intervenciones), la difusa asignación de roles dentro del equipo (que puede suponer que algunos profesio- nales realicen tareas para las que no están suficientemente acreditados), la ausencia de coordinación entre los diferentes modelos asistenciales que se están implementando (por cuestiones ideológicas o de conflictos de poder en los equipos) y la falta de criterios o criterios inadecuados como la presión asistencial, la disponibilidad en un momento dado o motivos personales - en la asignación de un terapeuta que asuma el grueso de la relación terapéutica y establezca con el sujeto las medidas a emplear y la disponibilidad de las mismas.

El trabajo en equipo, además, puede dificultar el proceso de información al paciente/usuario sobre el plan de tratamiento que se va a llevar a cabo.

\section{Los fines y los medios en la asistencia al drogode- pendiente}

A lo comentado se une otro aspecto de indudable valor para el análisis bioético, y es la reflexión sobre los fines y los medios del tratamiento, cuya definición resulta inexcusable para que pueda realizarse una "indicación" de una determinada terapia. No cabe duda de que los fines van a condicionar los medios que la sociedad ponga a disposición de los profesionales.

En cuanto a los fines, durante años tanto la sociedad como los profesionales han entendido que en el caso de las drogas legales bastaba una tolerancia paternalista, entendiendo que sólo era exigible la abstinencia cuando se producían problemas serios en el entorno derivados del consumo (por ejemplo en el caso del alcohol o las benzodiacepinas) y aún ello de forma comprensiva para las continuas recaídas, tan habituales en los adictos. Para las drogas ilegales, sin embargo, sólo cabía una opción, la abstinencia, como requisito para volver a formar parte del tejido social. Fines alternativos como la integración, la rehabilitación, la normalización, aún dentro del consumo, sólo pudieron plantearse desde la defensa social (ante las complicaciones planteadas por el SIDA, fundamentalmente) y con un sentido de "mal menor". Esta situación, afortunadamente parece estar revirtiendo(21), aunque lentamente, en los último años y la mayor parte de los profesionales que trabajan con drogodependientes en el ámbito asistencial pueden plantearse sin ningún tipo de reparo la coexistencia de ambos fines de forma no excluyente (ni en lo transversal ni longitudinalmente para un sujeto), sin que la finalidad paliativa sea contemplada como de segunda categoría(22,23).

En cuanto a los medios, el requisito imprescindible para que cualquier intervención sea considerada una forma de tratamiento es que pueda estar indicada, es decir, que haya demostrado su capacidad de incidir en el trastorno y modificarlo de acuerdo a los fines que comentábamos en el párrafo anterior. Así no caben intervenciones denominadas "terapéuticas" que no 
hayan sido probadas y demostradas, estableciéndose claramente los criterios de indicación. Esto resulta un problema de primera magnitud para las técnicas no estrictamente farmacológicas, que deben acostumbrarse a demostrar su efectividad de forma fehaciente (y no por opinión subjetiva o experiencia personal) antes de ser recomendadas. La indicación de una técnica no probada entra de lleno en conflicto con el principio de no-maleficencia.

El segundo criterio que un tratamiento debe cumplir para ser indicado es que la relación coste-beneficio sea adecuada. En un sistema de salud pública como el que disfrutamos o padecemos, donde las necesidades son ilimitadas y los recursos escasos, no cumple requisitos de justicia que se ofrezca en el catálogo de prestaciones un fármaco o una técnica que no haya realizado un buen balance de los riesgos que su uso produce frente a los beneficios probados, cabe en cualquier caso que un sujeto utilice sus propio medios económicos (probablemente en el ámbito de lo privado) para conseguir ese tipo de tratamiento o terapia. También es un serio problema de justicia el que no se ofrezca, por razones no terapéuticas, un fármaco o una técnica que haya probado su efectividad y cuyo balance riesgo-beneficio sea adecuado(24).

Finalmente el tratamiento debe cumplir un tercer requisito, que es el de la aceptación por parte de aquel que va a seguirlo. No cabe vulnerar la autonomía del paciente para elegir, entre las técnicas disponibles, aquella que él considera más acorde a su propio proyecto vital, presionando su elección o sustrayendo información relevante. Sólo, como veremos en otro apartado, cuando el sujeto no puede optar (y la capacidad para ello, como la inocencia en el ámbito jurídico o el valor en el ejército, "se le supone") habrá que determinar el titular de las decisiones de sustitución, lo que nunca puede ser óbice para no informar al sujeto sobre el tratamiento que se va a aplicar y las razones que han motivado la elección entre las diferentes alternativas disponibles.

Como vemos la perspectiva bioética puede y debe entrar en el análisis de hechos como la falta de intervención sanitaria y social sobre una adicción como la nicotina (situación que parece empezar a revertirse en los últimos años por influencia anglosajona), o como el alcohol (probablemente en nuestro país por los importantes intereses económicos); o como la aceptación de que pueda hacerse la deshabituación con la propia sustancia adictiva o no en dependencia de la sustancia de que se trate, criterio que a priori poco parece tener que ver con aspectos sanitarios; o con las dificultades para conseguir ubicar las drogodependencias, cuando las consideramos un trastorno, al mismo nivel que el resto de las patologías, o definir claramente cuáles son los criterios asistenciales para tratar a los drogodependientes en centros específi- cos, situación que, sin embargo, no se generaliza en el territorio español(25).

Un problema específico que no podemos dejar de tratar cuando hablamos de medios en el abordaje de las drogodependencias es el que concierne a los programas con dependientes a opiáceos. Nuestro país ha sufrido como otros la oposición de programas libres de drogas (hasta hace no muchos años eran la única opción) y de programas de sustitución(26), así como la valoración social (y, a veces, profesional) de que los programas de reducción de daños podían facilitar el incremento de los drogodependientes(27).

Probablemente es hora de que la sociedad en su conjunto pueda conocer, en base a los estudios realizados a nivel nacional e internacional $(28,29,30)$ y a la experiencia asistencial en nuestro país, que los programas de mantenimiento representan una alternativa terapéutica real y adecuada cuando los programas libres de droga no son posibles.

En efecto diferentes estudios demuestran que los programas de mantenimiento con opíaceos mejoran la retención en tratamiento, disminuyen las conductas de riesgo y las sobredosis, disminuyen las tasas de seroconversión y las dosis de opioides concomitantes y, asociados a técnicas de reinserción, disminuyen la criminalidad (considerada por muchos un fenómeno funcional ligado a la ilegalidad de la sustancia) y favorecen la normalización en el funcionamiento personal y social del sujeto drogodependiente(31).

Por otra parte también es importante promocionar el conocimiento entre la población general de que los programas de reducción de daños ( programas de prevención de intercambio de jeringuillas, programas de sexo seguro", programas de venopunción higiénica, etc.) no sólo son un medio de protección de la sociedad en su conjunto frente a la amenaza del SIDA y otras enfermedades infecciosas (lo cual es un objetivo intrínsecamente injusto si se plantea como objetivo único o principal), sino que puede ayudar a mejorar la dignidad de las condiciones de vida de muchos dependientes(32).

Finalmente, y aunque sea citarlo únicamente, señalar los múltiples problemas éticos que subyacen en la investigación de los tratamientos con drogodependientes por la dificultad de establecer adecuadamente las condiciones de prueba (cómo realizar el doble ciego, cómo controlar la utilización de sustancias tóxicas o incluso "ilegales," cómo realizar el seguimiento, etc.) $(33,34)$.

\section{Algunas propuestas relacionadas con el tratamiento}

El tratamiento de los drogodependientes exige establecer adecuadamente las características y dotación de los dispositivos de abordaje, que deben estar dotados de personal formado, con una clara asignación de roles, aunque parece deseable que el drogo- 
dependiente pueda conocer y establecer una relación terapéutica con un terapeuta referente que coordine las actuaciones del resto de los profesionales implicados, a menudo muy diferentes, y canalizar la informaciones y los deseos del paciente.

Los profesionales implicados en el tratamiento a drogodependientes deben individualizar el plan de tratamiento en base a una indicación o indicaciones formuladas sobre los conocimiento probados de su eficacia, sobre un adecuado balance coste-beneficios y con el consentimiento de un paciente adecuadamente informado de las alternativas.

Parece el momento de plantear un modelo de tratamiento que permita y facilite la desintoxicación y la deshabituación, pero que haga accesibles los programas de reducción de riesgos (entre ellos los programas de mantenimiento con opiáceos) como paso intermedio o como tratamiento paliativo para aquellos drogodependientes que no puedan o no quieran seguir programas libres de droga, evitando discriminarlos por esta opción. Por otra parte parece importante que los profesionales puedan estudiar y establecer criterios sobre los aspectos biopsicosociales del trastorno, sin desresponsabilizar al adicto, sin despreocuparse de la propia dependencia y sin utilizar medidas de todo tipo no suficientemente probadas(35).

En definitiva el mejor tratamiento es el tratamiento individualizado para cada sustancia pero sobretodo para cada individuo, multifactorial, contrastado en sus resultados, asumible socialmente en sus costes y aceptado libremente por el sujeto $(7,10)$.

De la misma manera que hoy socialmente puede aceptarse una cierta dosis de discriminación positiva con la mujer para tratar de superar años de discriminación negativa, en la atención a los drogodependientes, y más aún cuando se trata de dependientes a opiáceos, el hecho de que sea un colectivo que ha vivido marginado social y asistencialmente, y que ha sufrido y sufre las mayores tasas de morbimortalidad por VIH, infecciones de todo tipo, sobredosis e intoxicaciones causadas por drogas sin ningún control de calidad, podamos plantearnos, al menos en el ámbito asistencial, la necesidad de diseñar estrategias de abordaje a todos los niveles que permitan atender las necesidades de estos sujetos y evitar cualquier tipo de desatención o discriminación sanitaria.

Por último una breve mención al hecho de que determinados colectivos afectados por las dependencias y en situación social claramente desfavorable (prostitutas, penados, etc.) deberían recibir atención específica por los profesionales con criterios que contemplen sus peculiaridades de entorno, de la misma forma que se impone reflexionar y articular medidas para el frecuente fenómeno de la politoxicomanía.

\section{Competencia y Decisiones de sustitución}

Competencia es un término sinónimo de capacidad, que en este texto utilizamos para designar aquel estado cognitivo y volitivo del ser humano que le permite, en el seno de las decisiones asistenciales, comprender la situación a la que se enfrenta y las consecuencias previsibles de cada uno de lo cursos de acción que se le ofrecen, para a continuación realizar la elección más acorde con su propio proyecto vital y su escala de valores. Por tanto la competencia o capacidad, va más allá de la capacidad legal y se hace equivalente a la capacidad de obrar referida a una decisión concreta y es un supuesto positivo que debe valorarse en cada situación de forma individual(36). Un ejemplo conocido que suele emplearse para ilustrar esta relatividad es el de un niño, que puede no tener competencia suficiente para negarse a un tratamiento antibiótico cuando padece una infección (en este caso su incompetencia tiene que ver con su incapacidad cognitiva para entender y asumir la implicaciones de ese no-tratamiento) pero sin embargo puede ser competente para elegir la vía de administración de dicho fármaco, siendo su gusto personal un criterio respetable si varias vía están disponibles.

La competencia permite el ejercicio de la autonomía, esto es, del autogobierno, también en las decisiones sanitarias, sin otro límite que la no-maleficencia (no podemos pedir a un profesional que aplique medidas contrarias a la lex artis) o la justicia (no podemos solicitar un acto que no esté incluido en el catálogo de prestaciones, a menos que estemos en el ámbito privado).

Para que la competencia pueda ejercerse el sujeto debe estar adecuadamente informado de su proceso patológico y de las alternativas de tratamiento que se le pueden aplicar, y encontrarse libre de coacciones internas significativas (trastornos intrínsecos de la competencia por afectación de las facultades volitivas o cognitivas) y sin coacciones externas.

El tema de la evaluación de la competencia resulta a menudo problemático si tenemos en cuenta que la competencia no se establece como un punto de corte sino que entre la incompetencia y la competencia no existe solución de continuidad y sólo podemos hablar de grados, y si a esta realidad añadimos el hecho de que los instrumentos de evaluación no resultan fáciles de aplicar en todos los casos(37).

En lo que concierne al tratamiento de sujetos drogodependientes la situación es todavía más confusa: ¿podemos hablar de abandonar libremente una adicción? ¿Un sujeto esclavizado por una sustancia puede considerarse competente para tomar decisiones que tienen que ver con ella? ¿Cómo afecta la sustancia al funcionamiento cognitivo de un sujeto y por tanto a su capacidad para la toma de decisiones sanitarias en 
relación a otros trastornos ligados o no a su dependencia?

Probablemente es el tema más difícil de lo que hemos planteado hasta ahora y sobre el que existe un menor nivel de conocimiento. En general estamos considerando que el drogodependiente debe acudir voluntariamente a tratamiento(38) y por tanto lo estamos declarando competente, aunque algunos autores alzan su voz para comentar que debería poder realizarse el tratamiento forzoso en algunos casos en los que el sujeto demuestra incompetencia franca para cuidar de sí mismo $(39,40)$. Frente a esto, el sistema sanitario está procediendo a tratar a adictos que eligen el tratamiento como medida de seguridad frente a la cárcel, lo que tiene indudables asomos de tratamiento forzoso y que en cualquier caso reconoce en la dependencia capacidad suficiente de afectar a la voluntad o a la capacidad cognitiva del sujeto, o a ambas, como para constituir una atenuante o una eximente de la responsabilidad penal. No puedo ni debo ir mas allá en un tema tan "inmaduro" desde el punto de vista teórico; baste afirmar la necesidad imperativa de reflexionar desde qué criterios (y de que tipo) estamos afirmando la competencia o incompetencia de los sujetos drogodependientes para evitar tomar posturas que bien afecten a su libertad de autogobierno o bien sean maleficentes desde el abandono a una persona incapaz de hecho.

Hasta que esta reflexión permita establecer mejor en qué manera las drogodependencias afectan a la libertad, sólo es posible hacer una valoración individual y aproximada, y maximizar las condiciones físicas y psíquicas del sujeto y la información sobre las alternativas posibles de tratamiento a fin de conseguir la mejor autonomía posible.

Como en otros casos, cuando el sujeto es incompetente, entran en el escenario las medidas de beneficencia. Estas consisten fundamentalmente en la necesidad de que un allegado tome por el sujeto las decisiones sanitarias necesarias para realizar un tratamiento. Este es otro de los problemas importantes que puede plantearse en drogodependientes graves o de larga evolución en los que a menudo se han roto los lazos familiares o en los que ningún familiar o allegado puede representar al sujeto en sus intereses y proyecto de vida personal. En la evolución de una drogodependencia, y sin entrar siquiera en ésta, se pueden producir diferentes situaciones médicas físicas y psíquicas, por intercurrencia de otros trastornos, que afectan claramente a la competencia del paciente. Hasta el momento el papel de "padre bueno", en ausencia de familiares, está siendo desempeñado por sanitarios y organizaciones e instituciones ligadas a la atención al drogodependiente. Cabe comentar también una profundización en los modos y criterios de sustitución, para que estas decisiones subrogadas se tomen con la mayor adecuación.

\section{EN RESUMEN...}

No cabe duda que los conflicto bioéticos que plantea la asistencia a los drogodependientes va mucho más allá de lo que hemos comentado: este texto no pretende otra cosa que un acercamiento teórico-práctico y una finalidad sensibilizadora a este tipo de perspectiva.

Tratando de resumir algunos conceptos importantes podemos afirmar que desde los principios de nomaleficencia y beneficencia, las estrategias sanitarias en la atención al drogodependiente deben implementar programas de utilidad demostrada en problemas detectados y jerarquizados, relativos a la propia dependencia (programas libres de droga y programas de mantenimiento con sustitutivos) y relativos a los problemas físicos, psíquicos y sociales derivados de su consumo (entre ellos los programas de reducción de riesgos). Desde el principio de justicia debe cuidarse el dar a cada sujeto lo que necesita sin discriminarle sanitariamente por no alcanzar las expectativas sociales, y, desde el principio de autonomía, respetando su individualidad y su proyecto vital. Se trata en definitiva de abordar al drogodependiente como un individuo social con sus derechos y responsabilidades, permitiendo su integración y su normalización.

Finalmente sólo cabe, siguiendo a Diego Gracia(35), recordar en el campo asistencial tres conceptos fundamentales: en primer lugar que el drogodependiente es una persona (recordemos el sistema de referencia formal), en segundo lugar que es necesario respetar el proyecto de vida y la idea del bien de cada cual (independientemente de que no estemos de acuerdo con ellos) y finalmente que la sociedad puede y debe imponer límites a la libertad de los individuos a fin de proteger el bien común y la justicia.

Sin obviar la dificultad de abordaje de un tema como el de las drogodependencias, sólo cabe un último apunte sobre la necesidad de reforzar las medidas de prevención.

\section{BIBLIOGRAFÍA}

1. ABEL I FABRE F: El Diálogo Bioético en la Perspectiva del Tercer Milenio. Discurso de ingreso en la Reial Acadèmia de Medicina de Catalunya. Barcelona, 9 de mayo de 1999.

2. GRACIA D: Fundamentos de Bioética. Madrid EUDEMA, 1989.

3. GRACIA D: Principios y metodología de la bioética. Quadern CAPs 1993; 19: 7-17.

4. BEAUCHAMP TL, CHILDRESS JL: Principios de Ética Biomédica. Barcelona, Masson, 1999. 
5. FERNÁNDEZ-CREHUET NAVAJAS J: Ética frente a bioética. Med Clin 1999; 112 (2): 64-66.

6. GRACIA D: Prólogo. Las Drogas a debate: Ética y Programas de Sustitución. Fundación Ciencias de la Salud. Ediciones Doce Calles SL, Madrid, 1999.

7. MARTIN J: La drogue, la loi, l'éthique et la réalité. Psychotropes, 1990; VI (1): 61-72.

8. ALMOND B: Drug use and abuse: the ethical issues. In: Cocaine: scientific and social dimensions. Wiley, Chinchester (Ciba Foundation Symposium) 1992: 277-293.

9. WEST R: Addiction, ethics and public policy. Addiction 1997; 92(9): 1061-1070.

10. TEN HAVE H: Drug addiction, society and health care ethics. In: Principles of Health Care Ethics (Gillon R, de.). John Wiley and Sons Ltd., 1994: pp 895-902.

11. LAMO DE ESPINOSA E: De nuevo sobre las drogas. Claves de razón práctica 1990; 3: 15-19.

12. CESONI M, ROBERT CN: L'interdit desintégrant. Psychotropes 1991; VI (3): 17-29.

13. BROWER KJ, BLOW FC, BERESFORD TP: Treatment implications of chemical dependency models: an integrative approach. Journal of Substance Abuse Treatment 1989; 6: 147-157.

14. SADLER, J.Z.; WIGGINS, O.P.; SCHWARTZ, M.A.: Philosophical Perspectives on Psychiatric Diagnostic Classification. Baltimore: John Hopkins University Press, 1995.

15. MONTAGNE M, PUGH CB, FINK JL: Testing for drug use, part 2: legal, social and ethical concerns. Am J Hosp Pharm 1988; 45: 1509-1522.

16. FORREST AR: Ethical aspects of workplace urine screening for drug abuse. Journal of Medical Ethics 1997; 23: 12-17.

17. RASKIN C: Análisis de la detección del consumo de drogas y de alcohol en el lugar de trabajo: cuestiones morales, éticas y jurídicas. Boletín de Estupefacientes 1993; XIV (2): 51-94.

18. BLOCH S, CHODOFF P, GREEN S: Psychiatric Ethics (3rd ed.). New York, Oxford University Press, 1999.

19. DE BEER D: Bélgique: les premiers pas d'une longue marche vers la déprohibition. Psychotropes 1992; VII (2): 75-79.

20. KANTZIAN EJ: La hipótesis de la automedicación en drogodependencias: un punto de partida para reducir los riesgos. En: Las Drogas a debate: Ética y Programas de Sustitución (Gracia D, ed.). Fundación Ciencias de la Salud. Ediciones Doce Calles SL, Madrid, 1999: pp 30-51.

21. ARGANDOÑA M: Visión de la OMS. En: Las Drogas a debate: Ética y Programas de Sustitución
(Gracia D, ed.). Fundación Ciencias de la Salud. Ediciones Doce Calles SL, Madrid, 1999: pp 122-133.

22. RAINFORTH J: Literature review: arguments for and against changing the avalaibility of opioids. Australian National University, NCEPH, 1991.

23. ABEIJON JA: El horizonte de las intervenciones sobre drogas: hacia una complementariedad de estrategias. En: Las Drogas a debate: Ética y Programas de Sustitución (Gracia D, ed.). Fundación Ciencias de la Salud. Ediciones Doce Calles SL, Madrid, 1999: pp 166-175.

24. FARRELL $M$, WARD J, MATTICK R, HALL W, STIMSON GV, DES JARLAIS D, GOSSOP M, STRANG J: Methadone maintenance treatment in opiate dependence: a review. BMJ 1994; 309: 9971001.

25. DE LA FUENTE HOZ L, BARRIO ANTA G: Control de los problemas de salud asociados al consumo de drogas en España: hacia un abordaje científico y priorizado. Gac Sanit 1996, 10: 255-260.

26. DUPONT RL: Violencia y drogas. Revista de Toxicomanías 1999; 18: 26-28.

27. KALINA E: Legalizar la existencia tóxica. Adicciones 1995, 7(2): 227-231.

28. RODRIGUEZ MARTOS A: Reflexiones sobre los programas de mantenimiento con metadona. Adicciones 1994; 6(4): 353-372.

29. APA Official Actions: Position statement on methadone maintenance treatment. Am J Psychiatry 1994; 151(5): 792-794.

30. CASAS M. Presente y futuro de los programas de mantenimiento con agonistas opiáceos (editorial). Adicciones 1995; 7(4): 411-422.

31. MÁRQUEZY,POO M: Modelos de intervención: disminución de riesgos y programas de mantenimiento. Psiquiatría Pública 1998; 1(6): 378-388.

32. COLOM J. Los programas de reducción de riesgos. En: Las Drogas a debate: Ética y Programas de Sustitución (Gracia D, ed.). Fundación Ciencias de la Salud. Ediciones Doce Calles SL, Madrid, 1999: pp 16-29.

33. BOLINDER G: Tobacco research funded by the tobacco industry; an ethical conflict. Addiction 1997; 92(9): 1071-1075.

34. OSTINI R, BAMMER G, DANCE PR, GOODIN RE: The ethics of experimental heroin maintenance. Journal of medical ethics 1993; 19: 175-182.

35. GRACIA D: Por una cultura de la responsabilidad en el tema de las drogas. En: Las Drogas a debate: Ética y Programas de Sustitución (Gracia D, ed.). Fundación Ciencias de la Salud. Ediciones Doce Calles SL, Madrid, 1999: pp 242-263.

36. SIMÓN P: La evaluación de la capacidad de los pacientes para tomar decisiones y sus problemas. 
En. Cuestiones de Bioética (Feito L, ed.). Madrid, Dykinson, 1997: 119-154.

37. DRANE JF: The many faces of competence. Hastings Center Report 1985; 15(2): 17-21.

38. BETRAND MA: L'inmoralité de la prohibition. Psychotropes 1989; VI(1-2): 15-21.

39. DE MIRANDA S: The ethics of statutory coertion in the treatment of chemical substance abuse / dependence. Med Law 1989; 7: 427-432.

40. MARLOWE DB, KIRBY KC, BONIESKTE LM, GLASS DJ, DODDS LD, HUSBAND SD, PLATT JJ, FESTINGER DS: Assessment of coercive and noncoercive pressures to enter drug abuse treatment. Drug and Alcohol Dependence 1996; 4: 77-84. 